\title{
Enhancement of 3D Character Animations Through the Use of Automatically Generated Guidelines Inspired by Traditional Art Concepts
}

\author{
Jose A. S. Fonseca*, Denis Kravtsov, Anargyros Sarafopoulos and Jian J. Zhang \\ NCCA, Bournemouth University, UK
}

\section{Introduction}

Effective communication through character animation depends on the recognition of the performed body expressions. The creation of the right body postures is crucial for character animation in the context of animated films and games, as it allows for conveying the right set of emotions to the viewer. Audience needs to be able to identify familiar features mainly based on their own experiences, which allows the viewer to relate and feel empathy to observed characters. It is, therefore, crucial for the animator to accurately create the right posture and expressive body motion, during the posing phase of the animation process.

Although Posing is not one of the 12 Principles of Animation [Lasseter 1987], its achievement is the over-arching guiding principle and is the result of the sum of some of those principles.

Analysis of the works of novice and experienced animators indicates that 3D computer animated characters often lack expressiveness in their motion and generally look unnatural.

There is a need for a new conceptual guideline to arrange the body parts into a meaningful pose, as well as for a way of providing a visual guidance to the animator indicating that specific character postures of the animation sequence require further improvement.

\section{Our Approach}

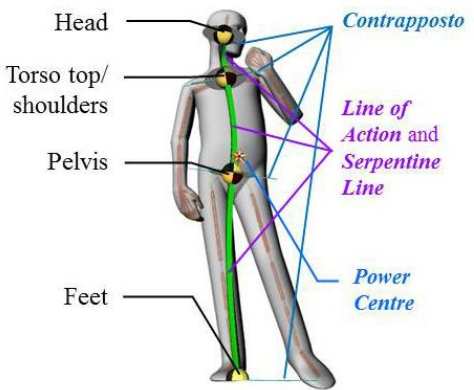

Figure 1: Basic relations between rig components and traditional art concepts

We suggest that certain concepts used in traditional animation and fine arts as well as acting, although often disregarded in computer animation, can be utilised for the enhancement of computer animated sequences through the improvement of key poses. We have identified four key concepts for creating a good and naturalistic pose (see Figure 1):

- Power Centre relates to the character's source of energy (see Figure 2) and has its roots in acting [Hooks 2011], being similar to the Chekhov's imaginary center [Chekhov 2010];

\footnotetext{
*e-mail: jfonseca@bournemouth.ac.uk
}

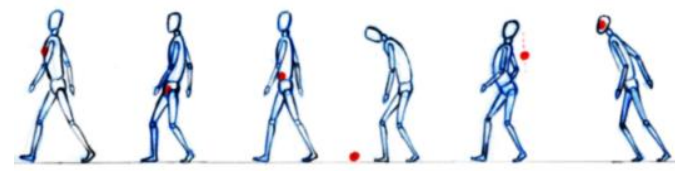

Figure 2: Examples of Power Centre

- Line of action is a line that assists in structuring the pose while drawing a figure for a specific action, according to the imaginary flow of energy going through the body (see Figure 3 ). This con-cept is rooted in traditional drawing techniques [Maestri 2006];

- Contrapposto is defined by the arrangement of the parts of the body in a bilateral opposition through the distribution of body weight between character's legs (see Figure 4). This concept was first developed by the Greek sculptor Polykleitos (5th century B.C.) [Pollitt 1995];

- Serpentine Line is an imaginary line (commonly S-shaped), which is also twisted in one direction, from the bottom to the top of the body [Shearman 1990]. It helps to structure the more dynamic postures of a figure (see Figure 5).

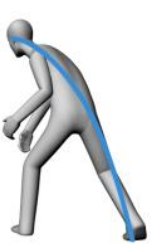

Figure 3: Example of Line of Action

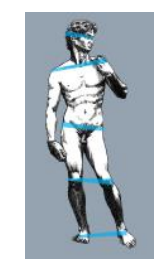

Figure 4: Example of Contrapposto ${ }^{1}$

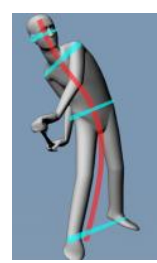

Figure 5: Example of Serpentine Line
The aforementioned concepts are synthesized in an imaginary line an expression cord - which represents the flow of energy through the body, structuring its main axis, and guiding the animator when dealing with the configuration of visual forces that are determinant to the creation of a specific pose. The most important parts crucial for the definition of the posture are: pelvis, shoulders, head and the position of the feet - centre of the base of support of the body (see Figure 1).

If the intended pose is static or there is little motion, the character has to be worked at least in terms of characterization, defining the power centre (which part of the body leads the posture) and corresponding posture as well as adjusting contrapposto, distributing the body weight in accordance to the intended expression. The animator has to think about the arrangement of the body parts in such a way that expresses the force that holds the body in that particular pose and which shape expresses a particular rhythm, that is synthesized by the expression cord. The feet should be placed in the convenient way to support the pose and the pelvis should be adjusted in different axes, according to the supporting foot, ensuring it is not aligned with the feet. The top of the torso needs to be adjusted in different axes, according to pelvis and making sure it is not aligned with the pelvis in the $\mathrm{Y}$ axis.

When creating dynamic pose, animator has to define the thrust or direction of the body and decide which part of the body leads it, according to the intended attitude to be portrayed. The shape of the body and the inherent arrangement of its parts are dependent on that 
thrust or source of energy and, therefore, so is the pose of the $3 \mathrm{D}$ character. In fact, all aforementioned actions of the animator follow the concept of line of action, along with the power centre, to orient the pose and define the inclination of the body. Next step is to consider position of the feet, either to respond to stability needs determined by the inclination of the body, or to express an unbalanced position. After that weight distribution, following the contrapposto, needs to be configured. The arrangement of body parts in a way to express the flow of energy going through the body - the expression cord - is complemented by the serpentine line rationale, producing a twist of the whole body and directing the head to the centre of interest.

It is apparent, that in order to achieve satisfying results animator has to consider a significant number of factors and a variety of concepts. It is rather easy to miss or forget one of them. We propose a system allowing for the improvement of the definition of the body posture and the resulting character animations. This is achieved through a high-level tool providing indication of the quality of the character pose, which allows to gain better under-standing of the rationale behind specific body expressions.

\section{Implementation}

Proposed approach was implemented as a set of plug-ins and scripts for Autodesk Maya $\odot$. Our conceptual system relies on the four notions we have outlined and allows to provide feedback to the animator in a simple and efficient way. It allows the animator to clearly understand a relationship between the effective pose and established high-level factors affecting the perception and the quality of the final animation.

The tool allows for an association between the joints or components of the rig of an arbitrary character with a minimal con-figuration setup. A relationship needs to be established between the components controlling the head, shoulders, pelvis, legs of the character and corresponding controls of the tool (see Figure 1). This is a simple process applied to the character's bind pose through a built-in UI. Once the relationship is established, visual guidance is automatically provided to the animator in real-time. Artist can therefore get immediate feedback during the posing phase and instantly amend key poses.

Once configured, an animator can define specific key poses paying attention to the expression cord. Modification of the character's pose is reflected in the change of the configuration of the cord as well as the colour of its segments. Initially, all curve segments are red (see Figure 6-a). As long as some segments remain red, orange or yellow, animator needs to improve corresponding part of the body of the character. For instance, in Figure 6-d curves and twists need to be improved. When the cord corresponds to a more natural and expressive pose, all segments change colour to green (see Figure 7). Additionally, animator always has an indication of the expression cord and its dynamics during the animation sequence. This gives a better higher-level understanding of the expressiveness of specific body postures comprising the final animation.

This way animator does not need to keep in mind all the factors affecting quality of the pose, rather focusing on setting the desired pose satisfying all conditions considered and hinted by our system.

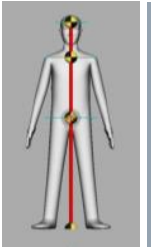

(a)

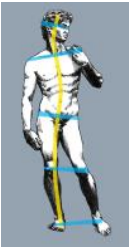

(b)

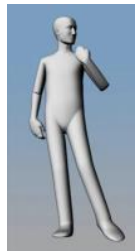

(c)

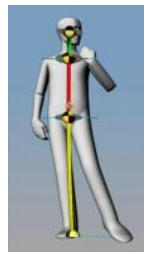

(d)

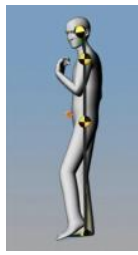

(e)
Figure 6: Tool in action: (a) bind pose, (b) reference pose ${ }^{1}$, (c) bad match of David's pose, $(d$, e) problematic areas revealed through the $3 D$ Pose Tool

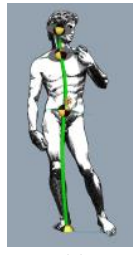

(a)

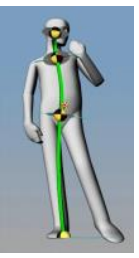

(b)

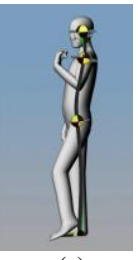

(c)
Figure 7: Tool in action: (a) David statue ${ }^{1}$ with a reference expression cord, (b, c) $3 D$ Character in the David's body posture

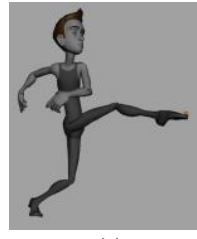

(a)

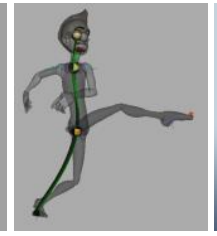

(b)

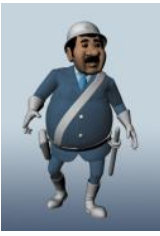

(c)

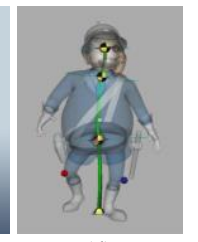

(d)
Figure 8: Body posture of different animated characters ${ }^{2}-(a)$ Pose Tool is off, (b) Pose Tool is on

\section{Conclusions}

We have proposed a new approach, as a conceptual guidance, allowing for assistance to novice and experienced animators in the creation of higher quality and more expressive character animations. We have implemented our approach in a popular tool used in production and educational environment, demonstrating that it has got real potential for use in existing and future pipelines without major effort. Furthermore, we strongly believe that the developed tool would be beneficial for improving the quality of computer animated sequences and saving time for artists of various levels. We are planning to perform further critical evalu-ation of the developed tool in the production and teaching process.

\section{Acknowledgements}

${ }^{1}$ Sketch after Michelangelo's David, by Jose Fonseca, amended with Contrapposto marks (Figure 4 and Figure 6-b) and with Pose Tool (Figure 7a).

${ }^{2}$ Morpheus character (Figure 8-a and b) used with permission of Josh Burton (www.joshburton.com).

Research funded by the Portuguese Foundation for Science and Technology (FCT) - PhD grant Ref.: SFRH/BD/61782/2009.

\section{References}

CheKhov, M., 2010. To the Actor. Oxon: Routledge.

HOOKS, E., 2011. Acting for Animators. $3^{\text {rd }}$ ed. Oxon: Routledge.

LASSETER, J., 1987. Principles of traditional animation applied to computer animation. Computer Graphics 21, 4 (July 1987), 35-44.

MAESTRI, G., 2006. Digital Character Animation 3. Berkeley: New Riders.

PollitT, J., 1995. The Canon of Polykleitos and Other Canons. In: Moon, W., ed. Polykleitos, the Doryphoros, and Tradition. Madison, Wis.: The University of Wisconsin Press, 19-24.

SHEARMAN, J., 1990. Mannerism. London: Penguin Books. 